\title{
LA RÉPARTITION ÉCOLOGIQUE DES AMPHIPODES DE LA FAMILLE DES GAMMARIDÉS DANS LES DÉPARTEMENTS FRANÇAIS DE L'HÉRAULT ET DES BOUCHES-DU-RHÔNE
}

\author{
par \\ MARION J. VAN MAREN \\ Institut de Zoologie taxonomique, Université d'Amsterdam, Pays-Bas
}

\begin{abstract}
The distribution and the autecology of 10 species of the amphipod family Gammaridae in fresh and brackish waters in south-eastern France (départements Hérault and Bouches-du-Rhône) are briefly described.
\end{abstract}

\section{INTRODUCTION}

$\mathrm{Au}$ cours des mois de juillet et août 1970 nous avons effectué des prospections systématiques dans les départements français de l'Hérault et des Bouches-du-Rhône. Sur les 260 stations visitées les 60 qui contenaient des Gammares ont été portées sur la figure 1 .

Dans toutes les stations à Gammares on a mesuré le taux en ions $\mathrm{Ca}$ et $\mathrm{Cl}$ et qualifié le biotope (largeur et profondeur des eaux, température, $\mathrm{pH}$, intensité du courant, estimation du degré de pollution, nature du fond et végétation).

\section{DESCRIPTION SOMMAIRE DE LA REGION PROSPECTEE}

Dans le département de l'Hérault des échantillons furent prélevés dans les rivières de l'Hérault et du Lez ainsi que dans leurs affluents. Ensuite quelques étangs de type lagunaire furent visités, tels que l'Etang de Berre et l'Etang de Vaccarès dans le département des Bouches-du-Rhône. De plus des échantillons accidentels furent recueillis dans des rivières situées dans la région entre Béziers et Marseille. Le tableau I donne un aperçu de la position des stations à Gammares. Les échantillons prélevés dans l'Hérault et dans ses affluents sont indiqués par $\mathbf{H}$, ceux du bassin du Lez par L, les autres par X.

On s'est servi des cartes Michelin 83 et 84 (échelle 1 : 200.000).

\section{a) Le bassin de l'Hérault}

L'Hérault prend son origine sur le Mont Aigoual (Cévennes) et se confond avec le Vis près de Ganges. Ici la rivière possède encore le caractère d'un ruisseau: bien qu'assez large elle contient de l'eau pure qui s'écoule à grande vitesse. Le fond est formé de sable et de cailloux. Près de Vissec l'affluent, le Vis, était complètement à sec. Le Lamalou contenait encore de l'eau, contrairement aux ruisseaux qui s'y jettent. Jusqu'à St. Jean-de Fos environ, l'Hérault garde les caractéristiques d'un ruisseau. Le paysage - qui est montagneux et boisé jusqu'ici - devient plat et sans végétation, la nature de la rivière change également. Aux environs de Gignac l'Hérault s'écoule encore à une vitesse modérée, mais possède une profondeur plus grande et un fond de pierres et d'argile. A l'ouest de Gignac se trouve un lac de barrage, le lac Salagou, appartenant aussi au bassin de l'Hérault. Beaucoup d'endroits au sud de Gignac dans le bassin de la rivière mentionnée ci-dessus étaient presque ou complètement à sec.

\section{b) Le bassin du Lez}

Le Lez prend sa source à $11 \mathrm{~km}$ environ au nord de Montpellier. Près de la source se trouve une installation pour l'alimentation en eau de cette ville.

L'affluent, le Terrieu, était en majeure partie à sec. A Prades-le-Lez, le Lez court encore à une vitesse assez grande, mais près de Montferrier le courant est déjà beaucoup plus lent. A cet endroit on ne peut pas atteindre le fleuve, à cause de la raideur des versants de la gorge. En aval de Montpellier le Lez est très pollué.

\section{c) Les autres stations d'échantillonnage}

La plupart des autres stations se situent dans les étangs au bord de la mer entre Béziers et Marseille. De plus, des échantillons furent prélevés dans quelques rivières qui s'y jettent, comme, par 
exemple, le Salaison. Ensuite plusieurs endroits ont été examinés dans l'eau principalement saumâtre du delta du Rhône, dont les marais sont drainés en grande partie par la construction de digues et de canaux. Outre ce vaste système de canaux il y a dans cette région beaucoup d'étangs dont l'eau est sujette à une évaporation considérable pendant l'été.

L'Etang de Berre à côté de Marseille communique avec la mer par le canal souterrain du Rove et par le Canal de Garonte. Surtout au nord un certain nombre de rivières se jettent dans l'étang, tels que le Touloubre.

\section{METHODES}

A chaque station on a prélevé des Gammaridés à l'aide d'une épuisette. Ensuite la température de l'eau fut mesurée à l'aide d'un thermomètre à mercure, et le $\mathrm{pH}$ fut déterminé approximativement à l'aide de papier à $\mathrm{pH}$. Un échantillon d'eau fut recueilli à chaque station; au laboratoire on en déterminait le taux de $\mathrm{Ca}$ avec une méthode de titrage spectrographique, à l'aide de l'"Oxford Titrator". La concentration en ions $\mathrm{Cl}$ fut déterminée électriquement avec un chlorinimètre E.E.L. On a noté un certain nombre de données sur le milieu à chacune des stations.

\section{RESULTATS}

La figure 2 montre les espèces des Gammares qui furent rencontrées aux stations "positives" pendant l'été 1970 . On y a ajouté les résultats des échantillonnages mentionnés dans "A revision of the European species of the Gammarus locusta-

\section{TABLEAU I}

Position des stations qui contenaient des Gammares

\begin{tabular}{|c|c|c|c|}
\hline Station & Nom du ruisseau & Lieu le plus proche et position & Particularités \\
\hline H 1 & & Montoulieu. D 108. Transversale à Viéle. & $\begin{array}{l}\text { Poissons morts sur } \\
\text { lesquels des Gammares }\end{array}$ \\
\hline $\mathbf{H}$ & & Brissac. Bifurcation D 108/D 4 & \\
\hline H 3 & Vis/Hérault & Ganges. D 25. Confluence Vis et Hérault & $\begin{array}{l}\text { Système de courant } \\
\text { compliqué }\end{array}$ \\
\hline H 4 & & N.O. de St. Laurent le M., D 110. & \\
\hline H 5 & Lergue & Pégairolles. D 149e. Pont. & Réserve de poissons \\
\hline H 6 & & Lauroux. D 15le. Pont. & \\
\hline H 7 & & Parlatges. D 25. Pont dans le village. & \\
\hline $\begin{array}{ll}\mathbf{H} & 8 \\
\mathbf{H} & 9\end{array}$ & & $\begin{array}{l}\text { Les Plans. D 35e. Pont. } \\
\text { Campestre. D 157. Pont à l'est de Lodève. }\end{array}$ & \\
\hline H 10 & & Villecun. D 157e, direction Olmet. & \\
\hline H 11 & & 3 km S.O. de St. Privat. D 153. & \\
\hline H 12 & & St. Privat. D 153. Pont dans le village. & \\
\hline H 13 & & $\begin{array}{l}\text { Les Salces. D 153e, après bifurcation } \\
\text { D } 153 / D 153 e \text {. }\end{array}$ & \\
\hline H 14 & & Lagamas. D 4. Pont. & \\
\hline H 15 & & 4,1 km N.E. de Cambous. & \\
\hline H 16 & & $\begin{array}{l}\text { Le Bosc. D 144. Premier ruisseau à l'O. } \\
\text { du village. }\end{array}$ & \\
\hline H 17 & & Lavalette. D 157e. Pont. & 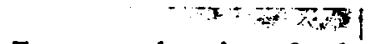 \\
\hline H 18 & & $\begin{array}{l}\text { St. Martin-des-C. D 157. Pont dans un } \\
\text { tournant }\end{array}$ & $\begin{array}{l}\text { Eau rouge-brunâtre, fond } \\
\text { d'argile rouge }\end{array}$ \\
\hline H 19 & & Clermont-l'H., direction Lac Salagou. & \\
\hline H 20 & Dourbie & Mourèze. D 8e. $1 \mathrm{~km} \mathrm{à} \mathrm{l'E.} \mathrm{du} \mathrm{village.}$ & \\
\hline H 21 & Peyne & N.D. de Mougères. D 174e, au S. du village. & \\
\hline H 22 & Rieutord & N.O. de Valros. D 125. & \\
\hline $\begin{array}{ll}\text { H } & 23 \\
\text { H } & 24\end{array}$ & Hérault & $\begin{array}{l}\text { Agde. D 32. 'Grau', } 2 \text { km au S. d'Agde. } \\
\text { N.E. de Gignac. D } 32 \text {. }\end{array}$ & \\
\hline H 25 & & Aniane. D 27. Pont en dehors du village. & \\
\hline $\begin{array}{l}\text { H } 26 \\
\text { H } 27\end{array}$ & Hérault & Causse-de-la-Selle. D 122. En amont du pont. & \\
\hline H 27 & Lamalou & Pont de Januc. D 122. 1,5 km à l'O. du village. & suivre d la page suivante \\
\hline
\end{tabular}




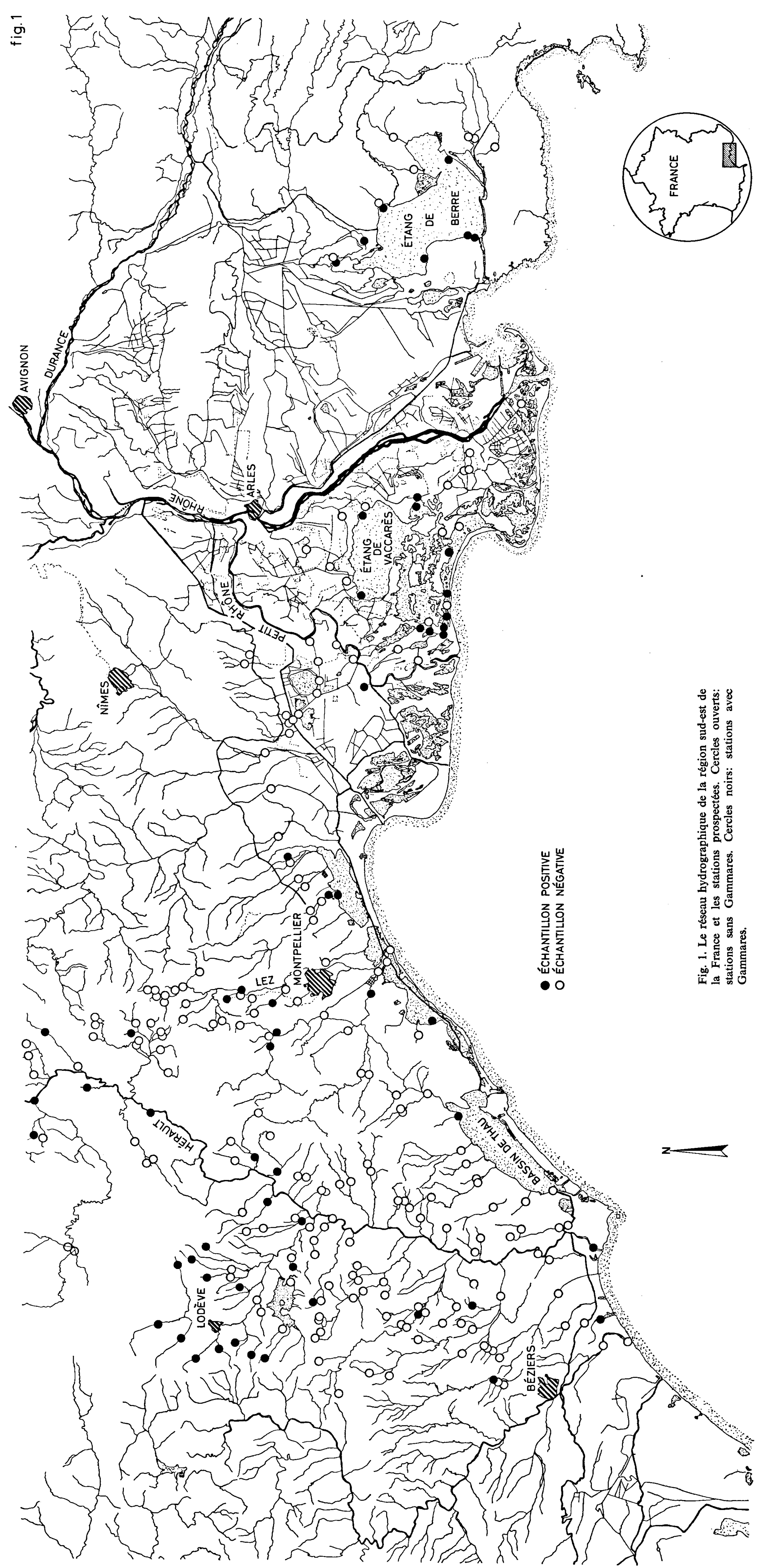



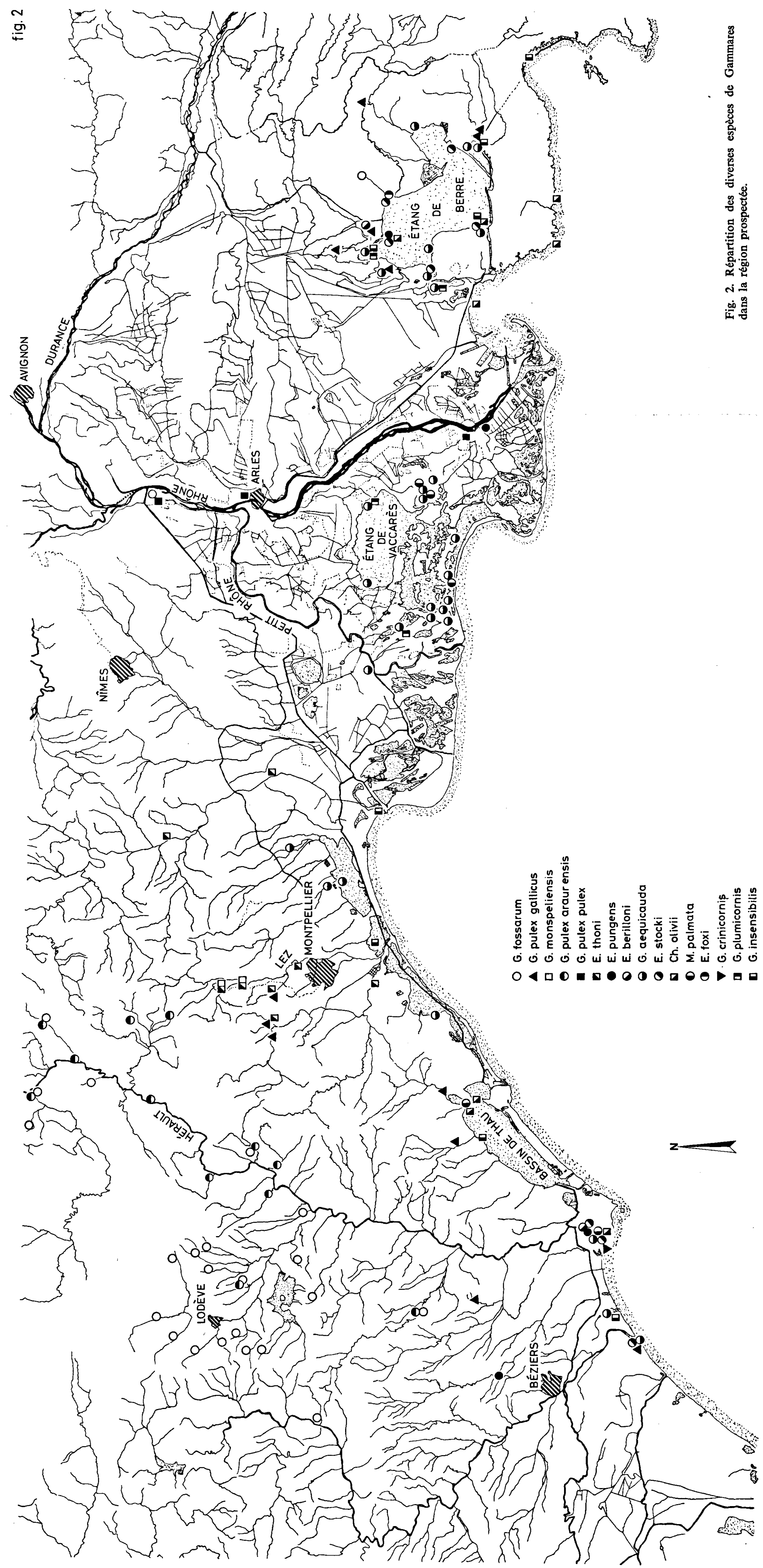
TABLEAU I, SUITE

\begin{tabular}{|c|c|c|}
\hline $\begin{array}{ll}\text { L } & 1 \\
\text { L } & 2 \\
\text { L } & 3 \\
\text { L } & 4 \\
\text { L } & 5 \\
\text { L } & 6\end{array}$ & $\begin{array}{l}\text { Mosson } \\
\text { Mosson }\end{array}$ & $\begin{array}{l}\text { Prades-le-Lez. D } 145 . \text { Pont. } \\
\text { Source du Lez. D } 112 \text {. } \\
\text { O. de Montferrier. D } 127 \text { e. } \\
\text { Grabels. D } 127,2 \mathrm{~km} \text { au N.O. du village. } \\
\text { S.E. de Combaillaux. D } 127 \text {. } \\
\text { Villeneuve-les-Mosson. D } 116 .\end{array}$ \\
\hline $\begin{array}{lr}X & 1 \\
X & 2 \\
X & 3 \\
X & 4 \\
X & 5 \\
X & 6 \\
X & 7 \\
X & 8 \\
X & 9 \\
X & 10 \\
X & 11 \\
X & 12 \\
X & \\
X & 13 \\
& \\
X & 14 \\
& \\
X & 15 \\
X & \\
X & 16 \\
X & \\
X & 17 \\
X & 18 \\
X & 19 \\
X & 20 \\
X & 21 \\
X & 22 \\
X & 23 \\
X & 24 \\
X & 25 \\
X & 26 \\
X & 27\end{array}$ & $\begin{array}{l}\text { Etang de Berre } \\
\text { Etang de Berre } \\
\text { Touloubre } \\
\text { Canal d'Istres } \\
\text { Etang de Berre } \\
\text { Etang de Berre } \\
\text { Etang de Vaccarès } \\
\text { Etang de Vaccarès } \\
\text { Etang de Vaccarès }\end{array}$ & 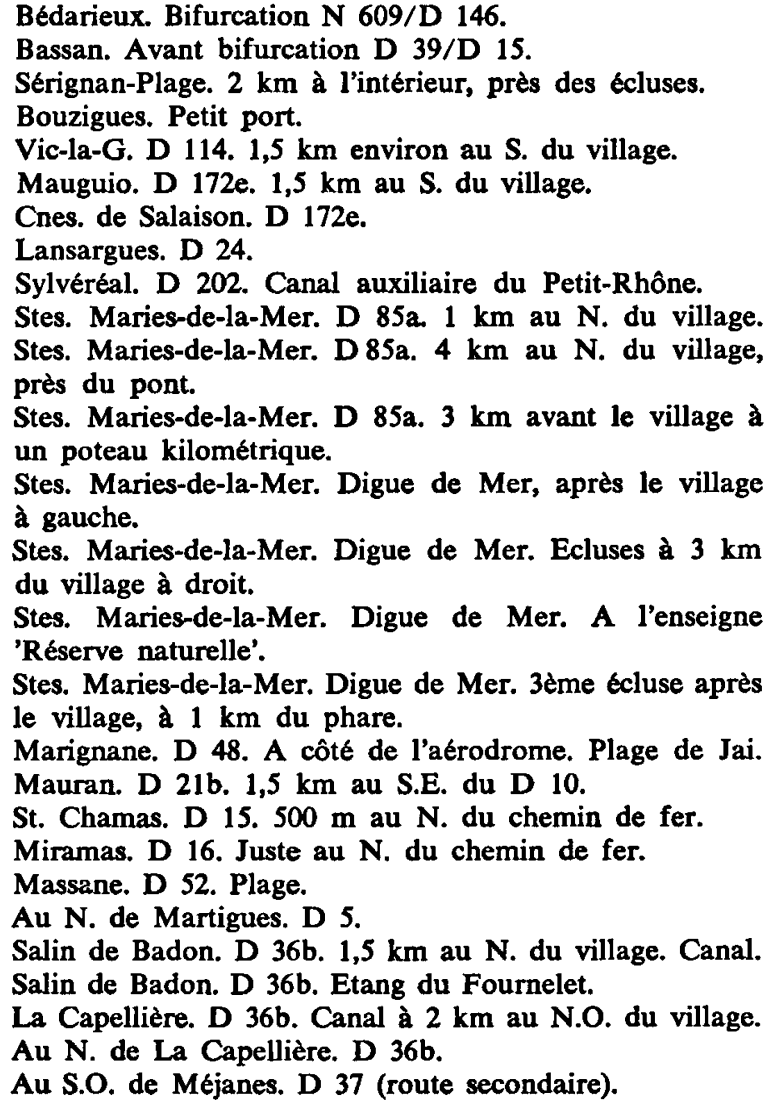 \\
\hline
\end{tabular}

group" (Stock, 1967) et "A revision of the European species of the Echinogammarus pungensgroup" (Stock, 1968).

Les espèces suivantes ont été rencontrées:

Gammarus fossarum Koch, 1835

G. pulex gallicus S. Karaman, 1929

G. pulex araurensis Pinkster, 1972

G. monspeliensis Pinkster, 1972

G. aequicauda (Martynov, 1931)

Echinogammarus thoni (Schäferna, 1922)

E. pungens (H. Milne Edwards, 1840)

E. stocki G. Karaman, 1970

Chaetogammarus olivii (H. Milne Edwards, 1830)

Melita palmata (Montagu, 1808).

Parmi les Gammares capturés en eau douce les espèces suivantes furent rencontrées le plus souvent: Gammarus fossarum, G. pulex gallicus et $G$. p. araurensis. Le nombre d'échantillons de
$G$, fossarum seul était suffisant pour en tirer des conclusions valables quant au milieu. Les figures 3-7 rendent compte du rapport entre les facteurs du milieu et la présence de $G$. fossarum et de deux autres Gammares. Les résultats suivants concernent exclusivement les stations où se trouvaient les espèces mentionnées ci-dessus:

1) Le degré de pollution (voir figure 3 et tableau II).

2) La teneur en ions $\mathrm{Ca}$ (tableau II et figure 4) variait de 35 jusqu'à $183 \mathrm{mg} / 1$ pour Gammarus fossarum, tandis que pour $G$. pulex gallicus des valeurs de 54-161 mg/1 furent relevées et pour G. p. araurensis on notait des valeurs de 43-149 $\mathrm{mg} \mathrm{Ca} / \mathrm{l}$.

3) La teneur en ions Cl (tableau II et figure 4) aux stations $\mathrm{H} 22$ et $\mathrm{H} 21$ était respectivement 
TABLEAU III

Les 6 stations "positives" dans le bassin du Lez

\begin{tabular}{|c|c|c|c|c|c|c|}
\hline Station & E. thoni & G. monspeliensis & G. p. gallicus & $\begin{array}{l}\text { Vitesse du } \\
\text { courant }\end{array}$ & Largeur $(m)$ & $\begin{array}{l}\text { Degré de } \\
\text { pollution }^{1} \text { ) }\end{array}$ \\
\hline $\begin{array}{l}\text { L } 1 \\
\text { L } 2 \text { (source) }\end{array}$ & $\begin{array}{l}\mathbf{x} \\
\mathbf{X}\end{array}$ & $\begin{array}{l}\mathbf{X} \\
\mathbf{X}\end{array}$ & $\overline{-}$ & rapide & 5 & $\begin{array}{l}\mathbf{X} \\
\mathbf{X}\end{array}$ \\
\hline L 3 & $\mathbf{x}$ & 一 & $\mathbf{x}$ & nul & 3 & $x \mathbf{x}$ \\
\hline L 4 & $\mathbf{X}$ & - & $\mathbf{X}$ & lent & & XX \\
\hline L 5 & - & - & $\mathbf{X}$ & lent & $0,5-1$ & $\mathbf{X X X}$ \\
\hline L 6 & $\mathbf{x}$ & 一 & - & lent & 6 & $\mathbf{X X}$ \\
\hline
\end{tabular}

$\mathbf{X}=$ présent

$-=$ absent

112 et $61 \mathrm{mg} / \mathrm{l}$ : des titres considérablement plus élevés que ceux des autres localités de la partie douce du bassin de l'Hérault.

4) Pour toutes les stations on notait un $\mathrm{pH} 6$.

5) La température. Voir fig. 5 et tableau II.

6) La vitesse du courant. Voir fig. 6 et tableau II.

7) La profondeur. Voir tableau II.

8) La largeur. Voir fig. 7 et tableau II.

9) Le fond (tableau II) de toutes les stations était constitué de cailloux, sauf à $H 18$, où une vase rougeâtre couvrait le fond. En presque $60 \%$ des localités il y avait également du sable.

En dehors des espèces mentionnées ci-dessus deux autres Gammares d'eau douce furent rencontrés: Echinogammarus thoni et Gammarus monspeliensis. Ils ont été capturés exclusivement dans le bassin du Lez. Le tableau III donne un aperçu des différents Gammares, du degré de pollution, de la rapidité du courant, et de la largeur de la rivière aux 6 stations du bassin du Lez. La température variait entre 18 et $20^{\circ} \mathrm{C}$ aux stations $\mathrm{L} 1-\mathrm{L} \mathrm{5}$, tandis qu'à $\mathrm{L} 6$ (dans l'affluent le Mosson) elle s'élevait à $33,8^{\circ} \mathrm{C}$. Le $\mathrm{pH}$ était 6 pour les échantillons du Lez. L'eau des stations L 1-L 5 titrait $36 \mathrm{mg} \mathrm{Cl} / 1$ en moyenne (la partie douce du bassin de l'Hérault donnait un titre moyen de $16 \mathrm{mg} \mathrm{Cl} / \mathrm{l})$. La station dans le cours inférieur du Mosson l'emportait de beaucoup sur les autres 5 localités avec une teneur en $\mathrm{Cl}$ de $142 \mathrm{mg} / \mathrm{l}$. Le fond de L 1, L 3, L 4 et L 5 est couvert de pierres, celui de L 6 de sable et celui de $L 2$ de vase et de sable. La profondeur des ruisseaux de toutes les stations visitées dans le bassin du Lez était inférieure à $0,50 \mathrm{~m}$.

Dans un échantillon originaire du Libron (X 2) se trouvait Echinogammarus pungens. La station hébergeant cette espèce fut caractérisée par les valeurs suivantes des différents facteurs du
1) $\mathrm{X}=$ propre; $\mathrm{XX}=$ légèrement pollué; $\mathrm{XXX}=$ assez pollué

milieu: une teneur en $\mathrm{Ca}$ de $107 \mathrm{mg} / \mathrm{l}$, un titre de $\mathrm{Cl}$ de $65 \mathrm{mg} / 1$ et une température de $20,6^{\circ} \mathrm{C}$. $A$ cet endroit la rivière s'écoulait à une vitesse modérée et l'eau était pure. Le fond était composé de gravier, la largeur était de $3 \mathrm{~m}$ et la profondeur $0,05 \mathrm{~m}$.

Gammarus aequicauda est le plus abondant parmi les espèces saumâtres (figure 2). Aux stations où ce Gammare était présent, le $\mathrm{pH}$ dépassait toujours 6 . La valeur le plus souvent constatée pour le $\mathrm{pH}$ s'élevait à 6,5 , mais à $X 5$ le $\mathrm{pH}$ montait même à 8,8 . La teneur en $\mathrm{Ca}$ (fig. 8) variait de $73 \mathrm{mg} / \mathrm{l}$ jusqu'à $525 \mathrm{mg} / \mathrm{l}$ (X 7, Etang de Mauguio) pour $G$. aequicauda. Le titre minimum de $\mathrm{Cl}$ s'élevait à $700 \mathrm{mg} / \mathrm{l}$ (dans un canal communiquant avec l'Etang de Vaccarès) et le maximum à $44200 \mathrm{mg} \mathrm{Cl} / \mathrm{l}$ (près de Stes. Maries-dela Mer). Aux stations où se trouvait l'espèce mentionnée ci-dessus le fond se composait le plus souvent de vase, parfois aussi de cailloux et de sable. La température variait entre 17,4 et $30,0^{\circ}$ $C$, tandis que la moyenne de $26^{\circ}$ est nettement plus élevée que celle des échantillons d'eau douce. Partout la profondeur dans les étangs était inférieure à $0,50 \mathrm{~m}$. Dans les autres localités celleci dépassait souvent $1 \mathrm{~m}$, tandis que la largeur des ruisseaux et des canaux dépassait toujours 1,50 m. Les échantillons qui ne proviennent pas des étangs ont été prélevés dans des cours d'eau lents ou, dans un seul cas, à courant modéré. Dans l'Etang de Berre (X 21) G. aequicauda se trouvait dans les brisants du lac. A X 23 l'eau était légèrement polluée, tandis qu'à $X 7, X 21$ et X 16 elle était pure. A toutes les autres stations où se trouvait ce Gammare le degré de pollution était d'importance négligeable.

Outre G. aequicauda trois autres espèces ont été capturées en eau saumâtre: dans 5 stations 


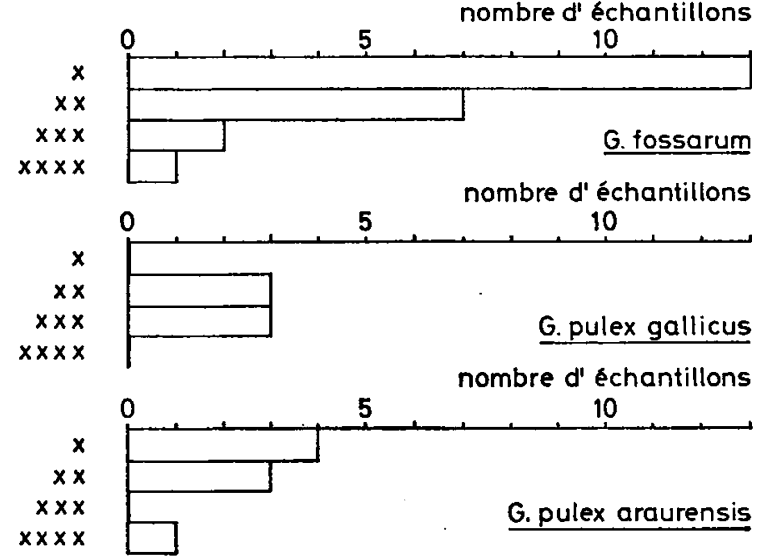

Fig. 3. Degré de pollution dans le milieu de Gammarus fossarum (en haut), G. pulex gallicus (centre) et G. pulex araurensis (en bas).

$\mathbf{X}=$ propre; $\mathbf{X X}=$ légèrement pollué; $\mathbf{X X X}=$ assez pollué; $\mathbf{X X X X}=$ pollué.

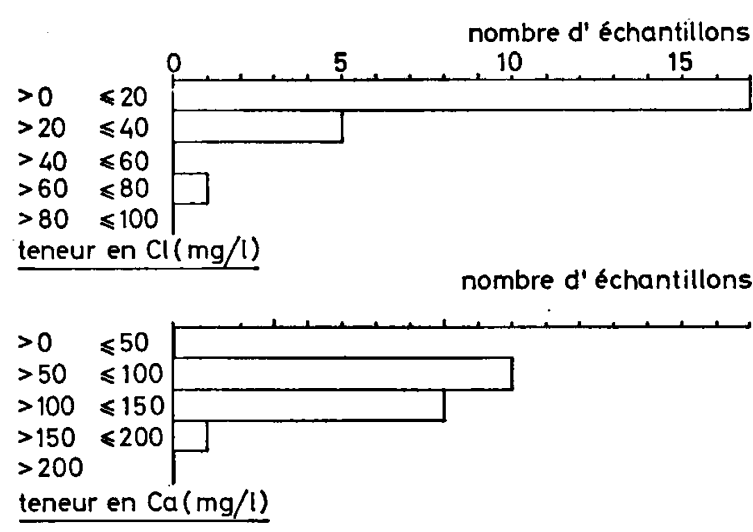

Fig. 4. Analyses du milieu de Gammarus fossarum: teneur en $\mathrm{Cl}$ (en haut) et en $\mathrm{Ca}$ (en bas).

nombre d' echantillons

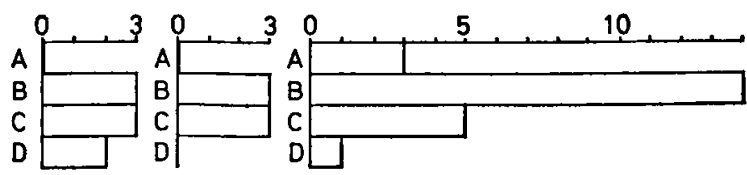

Fig. 5. Températures dans le milieu de Gammarus fossarum (à droit), G. pulex gallicus (centre) et G. pulex araurensis (à gauche).

$\mathrm{A}=>10^{\circ}$ et $\leqslant 15^{\circ} \mathrm{C} ; \mathrm{B}=>15^{\circ}$ et $\leqslant 20^{\circ} \mathrm{C} ; \mathrm{C}=$ $>20^{\circ}$ et $\leqslant 25^{\circ} \mathrm{C} ; \mathrm{D}=>25^{\circ}$ et $\leqslant 30^{\circ} \mathrm{C}$.
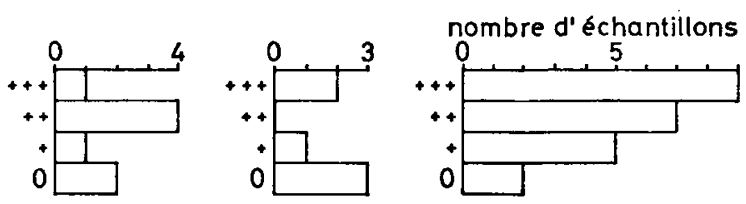

Fig. 6. Vitesse du courant dans le milieu de Gammarus fossarum (à droit), G. pulex gallicus (centre) et G. pulex araurensis (à gauche).

$+++=$ vif; $++=$ moyen; $t=$ lent; $0=$ nul.
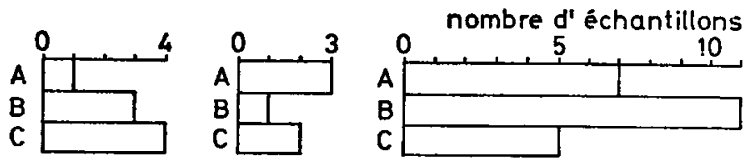

Fig. 7. Largeur du cours d'eau, aux stations à Gammarus fossarum (à droit), G. pulex gallicus (centre) et G. pulex araurensis (à gauche).

$A=\leqslant 1 \mathrm{~m} ; \mathrm{B}=>1 \mathrm{~m}$ et $\leqslant 2 \mathrm{~m} ; \mathrm{C}=>2 \mathrm{~m}$.

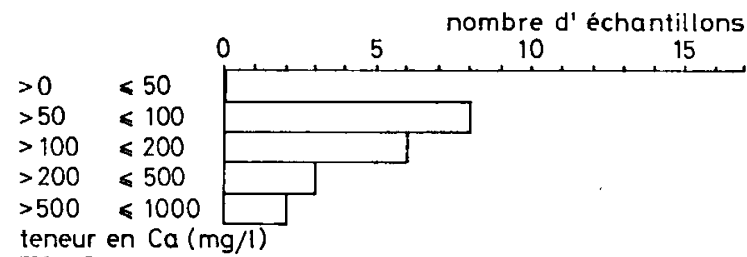

nombre d' échantillons

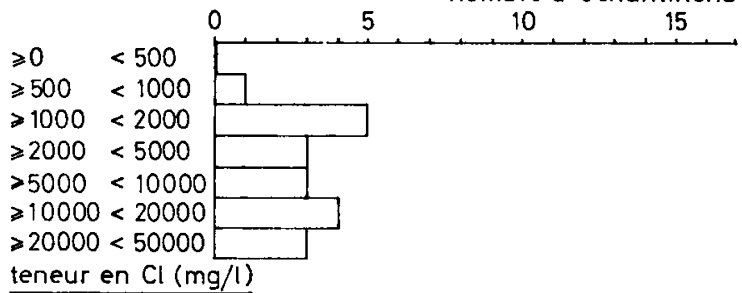

Fig. 8. Teneur en $\mathrm{Ca}$ (en haut) et en $\mathrm{Cl}$ (en bas) dans le milieu de Gammarus aequicauda.

Echinogammarus stocki et dans une station Chaetogammarus olivii était présent. Le tableau IV donne un aperçu des conditions de milieu dans les localités à Melita palmata et Echinogammarus stocki. La profondeur aux endroits où la première espèce fut rencontrée, ne dépassait pas $0,30 \mathrm{~m}$, sauf à $X 23$ où la profondeur dépassait $1 \mathrm{~m}$. Partout Melita palmata fut rencontré avec $G$. aequicauda, sauf dans le Bassin de Thau (X 4) où ce dernier n'était pas présent, mais où Chaetogammarus olivii fut rencontré.

Les stations à Echinogammarus stocki sont toutes situées dans l'Etang de Berre. La profondeur de l'eau à ces trois endroits était $0,20 \mathrm{~m}$. 


\section{TABLEAU IV}

A) Facteurs du milieu aux stations à Melita palmata.

B) Facteurs du milieu aux stations à Echinogammarus stocki.

\begin{tabular}{|c|c|c|c|c|c|c|c|c|}
\hline & Station & Temp. $\left({ }^{\circ} \mathrm{C}\right)$ & $p H$ & $\mathrm{Ca}(\mathrm{mg} / \mathrm{l})$ & $\mathrm{Cl}(\mathrm{mg} / \mathrm{l})$ & Fond $^{1}$ ) & $\begin{array}{l}\text { Vitesse du } \\
\text { courant }\end{array}$ & $\begin{array}{l}\text { Dégré de } \\
\text { pollution }{ }^{2} \text { ) }\end{array}$ \\
\hline A) & $\begin{array}{lr}X & 24 \\
X & 4 \\
X & 5 \\
X & 15 \\
X & 23\end{array}$ & $\begin{array}{l}28,4 \\
25,4 \\
28,0 \\
26,4 \\
25,4\end{array}$ & $\begin{array}{l}6,5 \\
6,5 \\
8,8 \\
7,0 \\
6,5\end{array}$ & $\begin{array}{r}95 \\
326 \\
450 \\
625 \\
73\end{array}$ & $\begin{array}{r}2800 \\
21000 \\
21800 \\
33200 \\
700\end{array}$ & 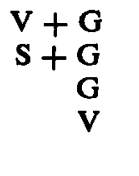 & $\begin{array}{l}\text { nul } \\
\text { nul } \\
\text { nul } \\
\text { nul } \\
\text { médiocre }\end{array}$ & $\begin{array}{l}\mathbf{X X} \\
\mathbf{X X} \\
\mathbf{X X} \\
\mathbf{X X} \\
\mathbf{X X X}\end{array}$ \\
\hline B) & $\begin{array}{ll}X & 17 \\
X & 18 \\
X & 22\end{array}$ & $\begin{array}{l}30,0 \\
24,0 \\
23,8\end{array}$ & $\begin{array}{l}6,5 \\
6,0 \\
6,5\end{array}$ & $\begin{array}{l}147 \\
120 \\
128\end{array}$ & $\begin{array}{l}6800 \\
1300 \\
7900\end{array}$ & $\begin{array}{l}G \\
G \\
G\end{array}$ & $\begin{array}{l}\text { nul } \\
\text { nul } \\
\text { nul }\end{array}$ & $\begin{array}{l}\mathbf{X} \\
\mathbf{X} \\
\mathbf{X}\end{array}$ \\
\hline
\end{tabular}

1) $\mathrm{G}=$ galets; $\mathrm{S}=$ sable; $\mathrm{V}=$ vase.

2) $\mathrm{X}=$ propre

$\mathrm{XX}=$ légèrement pollué

$\mathrm{XXX}=$ assez pollué

\section{DISCUSSION}

Gammarus fossarum fut trouvé le plus souvent en eau pure, tandis que Gammarus pulex gallicus se trouvait en eau peu ou moyennement polluée. Il n'est pas facile de donner une indication sur le degré de pollution du milieu de $G$. pulex araurensis puisque le nombre d'échantillons contenant cette espèce est faible, mais elle montre une préférence nette pour des eaux pas ou peu polluées. Le degré de pollution de la rivière augmente généralement à mesure qu'on descend plus en aval. Si l'on considère la répartition des Gammares mentionnés ci-dessus dans le bassin de l'Hérault, $G$. fossarum se trouve surtout dans la partie la plus en amont du courant principal et de ses affluents. Ce modèle de répartition est en accord avec les résultats trouvés dans la rivière le Slack (Pas-deCalais) par Stock, Nijssen \& Kant (1966). Gammarus pulex gallicus fut le plus souvent rencontré dans le cours inférieur de l'Hérault et de ses affluents, tandis que $G$. $p$. araurensis semble occuper une situation intermédiaire. Selon Nijssen (1963) G. fossarum se trouve aux Pays-Bas exclusivement en eau courante non-polluée et douce, à un $\mathrm{pH}$ de $6,2-7,0$ et il semble que la température, la composition du fond, la végétation et l'exposition au soleil ne soient pas des facteurs importants pour la présence de cette espéce et que la vitesse du courant ne joue pas un rôle important, à condition que l'eau ne soit pas stagnante. D'après les résultats disponibles il paraît que $G$. fossarum - contrairement par exemple à $\boldsymbol{G}$. pulex gallicus et à G. p. araurensis - atteint son optimum dans la région prospectée là où les tem- pératures ne dépassent pas $20,2^{\circ}$. Bien que $G$. fossarum ait été rencontré une fois en eau stagnante, il se trouvait pourtant le plus souvent dans des eaux à courant rapide. Des expériences de laboratoire exécutées par Roux (1967) démontraient que Gammarus fossarum est moins fertile et produit des juvéniles à vitalité plus réduite que $G$. pulex s.str., lorsqu'il vit en eau stagnante. (Roux démontrait par expériences d'hybridation que $G$. pulex gallicus et $G$. pulex s.str. font partie de la même entité génétique.) Selon l'auteur mentionné ci-dessus fossarum serait présent surtout aux stations dans lesquelles la température de l'eau reste relativement basse en été, et la répartition des Gammares serait limitée soit par le jeu des écarts thermiques soit par l'action des températures extrêmes. G. fossarum adapte son métabolisme respiratoire pour des températures jusqu'à $20^{\circ}$, au delà il réduit sa consommation d'oxygène. $G$. pulex gallicus par contre est capable d'adapter son métabolisme respiratoire jusqu'à une valeur de $25^{\circ} \mathrm{C}$. Pendant la recherche dont il est question ici cette espèce fut capturée le plus souvent en eaux à des températures entre 15 et $20^{\circ}$. La zone de $15-20^{\circ}$ est donnée par Roux comme zone optimale pour ce Gammare.

Les teneurs en $\mathrm{Ca}$, titrées aux stations à $G$. fossarum, s'accordent en majeure partie avec les résultats trouvés par Roux (1967), sur le terrain. Il démontrait cependant, avec des expériences au laboratoire, que les populations de fossarum vivant en eaux à des titres de Ca très différents appartiennent à des races physiologiques différentes. Dans le Midi de la France G. fossarum fut captu- 
ré surtout en eaux aux titres de 50 à $100 \mathrm{mg} \mathrm{Ca} / \mathrm{l}$, donc en eau "dure", dans la terminologie de Baldwin, 1967.

Aux localités à fossarum la teneur en $\mathrm{Cl}$ oscillait le plus souvent entre 0 et $20 \mathrm{mg} / \mathrm{l}$. Les valeurs élevées de $\mathrm{Cl}$ (et également de $\mathrm{Ca}$ ) aux stations $\mathrm{H} 21$ et $\mathrm{H} 22$ furent causées sans doute par l'évaporation considérable qui se produit dans cette région aride en été. Ces salinités élevées ne gênaient pas encore la présence des espèces d'eau douce mentionnées ci-dessus. L'absence de $G$. fossarum et $G$. p. araurensis à $\mathrm{H} 22$ ne démontre pas que ces deux Gammares ne puissent pas supporter la salinité en cet endroit. Stock, Nijssen \& Kant (1966) mentionnent la présence de fossarum, comme seul habitant, d'un ruisseau sur la plage près d'Ambleteuse-sur-Mer (Pas-de-Calais). Ils arrivent à la conclusion que la présence d'espèces compétitrices est pour $G$. fossarum le facteur limitant et non pas la salinité. Parce que G. pulex gallicus était abondant et que de nombreux individus étaient en précopulation à la sta-

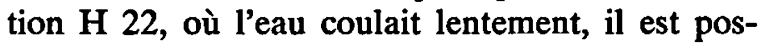
sible que la présence des deux autres Gammares ait été empêchée par une compétition trop forte. Il semble que $G$. fossarum et $G$. pulex gallicus se fassent concurrence l'un à l'autre à peu près comme l'ont constaté pour $G$. fossarum et $G$. pulex s.str. dans le bassin de la Slack Stock, Nijssen \& Kant, 1966. Dans le Midi de la France Gammarus pulex gallicus et $G$. p. araurensis ne furent jamais rencontrés à la même station, tandis que chacun de ces deux Gammares furent bien trouvés quelquefois ensemble avec $G$. fossarum (voir Pinkster, 1972).

Echinogammarus thoni et Gammarus monspeliensis sont présents dans le bassin du Lez. Tandis que la dernière espèce est limitée à cette rivière, Stock (1968) cite pour $E$. thoni encore deux localités: dans la rivière la Vidourle (dép. Hérault) et dans une source près de Balaruc-les-Bains. Comme milieu, pour ce Gammare, il mentionne entre autre la région de la source et le cours moyen des rivières; cette espèce est souvent associée avec des membres du groupe pulex. Ceci est en accord avec les résultats obtenus lors de la recherche actuelle: Echinogammarus thoni fut rencontré trois fois avec Gammarus pulex gallicus et deux fois avec $G$. monspeliensis et était présent surtout dans la partie supérieure du bassin du Lez et dans les sources de cette rivière. A la station L 5, qui était moyennement polluée, contrairement aux autres localités, $E$. thoni et $G$. monspe- liensis n'étaient pas présent, mais on y rencontrait Gammarus pulex gallicus. La station L 6 (dans le Mosson), où $E$. thoni était la seule espèce de Gammares présente, différait beaucoup des autres stations non seulement par la température $\left(33,8^{\circ}\right)$, mais également par sa teneur en $\mathrm{Ca}$ et $\mathrm{Cl}(142$ $\mathrm{mg} \mathrm{Cl} / \mathrm{l}$ ). Gammarus monspeliensis se trouvait exclusivement à la source et dans les zones à eaux pures et rapides du Lez.

Echinogammarus pungens, recueilli seulement dans la rivière le Libron, fut rencontré par Stock (1968) dans un ruisseau près de Balaruc-les-Bains, où nous ne l'avons pas retrouvé en 1970. De plus il mentionne la présence de cette espèce aux environs de plusieurs embouchures, comme celle de l'Hérault, du Rhône (près de Port-St. Louis) et du Touloubre (Etang de Berre). Harant \& Jarry (1963) citent en outre le Lez comme rivière dans laquelle ce Gammare fut capturé, mais très probablement il s'agit en ce cas de l'espèce indiquée en 1964 par Brun \& Brun comme "Gammarus pungens du Lez" et appelée Echinogammarus thoni aujourd'hui. Les titres de $\mathrm{Cl}$ donnés par Stock pour $E$. pungens varient de 20 à $3700 \mathrm{mg} / \mathrm{l}$, de sorte que la valeur trouvée dans le Libron de $65 \mathrm{mg} / 1$ s'inclut entre ces limites. Suivant Stock $E$. pungens est un Gammare d'eau saumâtre courante ou de grands lacs à teneurs élévées en ions (surtout de $\mathrm{Ca}$ ). Le titre de $\mathrm{Ca}$ à la station dans le Libron est plus bas qu'aux stations $\mathrm{H} 21$ et $\mathrm{H}$ 22 , où des représentants du groupe pulex furent rencontrés (la teneur en $\mathrm{Cl}$ de $\mathrm{X} 2$ était un peu supérieure à celle de $\mathrm{H} 21$ et plus basse que celle de la station $H$ 22). Les stations $H 21$ et $H 22$ se trouvent dans la même région que $\mathrm{X} 2$ mais dans un bassin différent. Il est possible que Gammarus pungens puisse pénétrer et se reproduire (des Gammares en précopulation furent capturés) si loin en amont dans le Libron à cause de l'absence de membres du groupe pulex et d'autres espèces compétitrices (voir Stock, 1968).

Les Gammares du groupe locusta sont reprèsentés surtout par Gammarus aequicauda dans les eaux saumâtres méditerranéennes. Kant et al. (1968) citent pour cette espèce des teneurs en $\mathrm{Cl}$ variant de 810 à $21300 \mathrm{mg} / \mathrm{l}$. Pendant la recherche de l'été 1970 un maximum de $44200 \mathrm{mg} / 1$ fut constaté (près de Stes. Maries-de-la Mer) et un minimum de $700 \mathrm{mg} \mathrm{Cl} / \mathrm{l}$. Ce dernier titre de $\mathrm{Cl}$ fut rencontré à l'endroit où un ruisseau se jetait dans un canal communiquant avec l'Etang de Vaccarès (X 23).

D'après Guigues (1961) des aequicauda juvéni- 
les ne survivent jamais - contrairement aux adultes - à une chlorinité de $20000 \mathrm{mg} / \mathrm{l}$. Elle mentionne pour $G$. aequicauda dans l'Etang de Vaccarès deux périodes de reproduction: à savoir le mois de mai et le mois de décembre. En 1970 des exemplaires d'aequicauda en précopulation furent rencontrés dans cet étang au début du mois d'août.

Selon Petit \& Schachter (1959) cet étang serait fortement adouci surtout par la décharge de l'eau venant des rizières. En 1970 des individus de $G$. aequicauda en précopulation furent trouvés, près de Méjanes, à des chlorinités allant jusqu'à 10100 $\mathrm{mg} / \mathrm{l}$. Ce Gammare fut capturé pendant la recherche dont il est question ici, surtout à des titres de Ca entre 50 et $100 \mathrm{mg} / 1$. A la station $X$ 10, près de Stes.-Maries-de-la Mer, à une chlorinité de $44200 \mathrm{mg} / \mathrm{l}$, où l'on trouvait $G$. aequicauda, la teneur en Ca ne s'élevait qu'à $88 \mathrm{mg} / \mathrm{l}$. Il est possible que cette espèce préfère une teneur de $\mathrm{Ca}$ relativement basse, tandis que le titre de $\mathrm{Cl}$ peut être très élevé sans devenir un facteur restrictif.

Melita palmata fut rencontré très souvent en association avec $G$. aequicauda. Melita palmata, espèce marine qui peut subir le contact avec l'eau saumâtre seulement pendant un certain temps (Stock, Nijssen \& Kant, 1966), se trouvait en eau à une teneur en $\mathrm{Cl}$ variant de 700 à 33200 $\mathrm{mg} / \mathrm{l}$. Au voisinage de $\mathrm{X} 23$ où la chlorinité était de $700 \mathrm{mg}$ (voir ci-dessus la description de cette station) l'étang fut visité également. Dans l'étang se trouvait également Melita palmata et la chlo- rinité était de $2800 \mathrm{mg} / \mathrm{l}$.

Echinogammarus stocki fut capturé en trois endroits dans l'Etang de Berre. Cette espèce fut signalée par Stock (1968), sous le nom d'E. acarinatus, entre autre de près de l'embouchure de l'Hérault. Il mentionne pour $E$. stocki des titres de $\mathbf{C l}$ variant de 100 à $18900 \mathrm{mg} / \mathrm{l}$. Les résultats de la présente recherche ne sont pas en contradiction avec ces données. Comme le signale Stock (1968) le substrat des localités à $E$. stocki consistait en gravier.

Chaetogammarus olivii fut rencontré dans le Bassin de Thau, à une teneur en $\mathrm{Cl}$ de 21000 $\mathrm{mg} / \mathrm{l}$. Ce titre de $\mathrm{Cl}$ est légèrement plus élevé que la valeur de $20500 \mathrm{mg} \mathrm{Cl} / 1$ mentionnée par Stock (1968) comme maximum pour cette espèce.

Nous n'avons pas trouvé de Gammares dans certaines zones prospectées. Dans la plupart des cas les ruisseaux visités étaient pendant l'été partiellement ou complètement à sec. Le lac Salagou (bassin de l'Hérault) est un exemple où l'aridité ne fut pas la cause de l'absence des Gammares, car dans ce lac de barrage se trouvent poissons et larves de moustiques (sans doute, le facteur écologique qui compte là, c'est le changement brusque dans le niveau d'eau).

Pour le reste un certain nombre de localités exemptes de Gammares étaient très polluées: un ruisseau aux environs de St. Laurent-le-Minier contenait une eau laiteuse chargée de déchets des mines. La rivière la Lergue, un affluent de l'Hérault, contenait - au sud de la ville de Lodève de l'eau très polluée et presque stagnante.

\section{REFERENCES BIBLIOGRAPHIQUES}

BALDWIN, E., 1967. Vergelijkende biochemie: 1-152. (Aula-boeken, Utrecht, Antwerpen).

BrUN, G. \& B. Brun, 1964. Sur la répartition et la taxonomie des Gammares du groupe Gammarus pungens dans le Sud-est de la France. Bull. Soc. zool. France, 89 : $754-759$.

Guigues, S., 1961. Morphologie et biologie de Gammarus (Gammarus) locusta L. de l'étang du Vaccarès. Ann. Fac. Sci. Marseille, 31 : 153-163.

Harant, H. \& D. JARRY, 1963. Guide du naturaliste dans le Midi de la France, 2: 1-369. (Delachaux \& Niestlé, Neuchâtel).

Kant, P., W. De LeeuW, S. Pinkster, A. E. Rijnberg \& J. H. STock, 1968. La répartition d'espèces de Gammaridae dans quelques étangs au nord de Banyulssur-Mer. Versl. zool. Werkexc. Banyuls-sur-Mer, 1968 : 3-21 (Zool. Mus. Amsterdam).

NIJSSEN, H., 1963. Some notes on the distribution and the ecology of the amphipod Gammarus fossarum Koch, 1835 , in the Netherlands (Crustacea Malacostraca). Beaufortia, 10 (116) : $40-43$.
Pettr, G. \& D. Schuchter, 1959. Les étangs et lagunes du littoral méditerranéen français et le problème de la classification des eaux saumâtres. Arch. Océanogr. Limn., 11 (Suppl.): 75-91.

Pinkster, S., 1972. On members of the Gammarus pulexgroup (Crustacea-Amphipoda) from western Europe. Bijdr. Dierk., 42 (2) : $164-191$.

Roux, A. L., 1967. Les Gammares du groupe pulex (Crustacés Amphipodes). Essai de systématique biologique. Thèse Fac. Sci. Univ. Lyon, 447 : I-VII, $1-172$.

Stock, J. H., H. NiJssen \& P. KaNT, 1966. La répartition des Amphipodes de la famille des Gammaridae dans la Slack et son estuaire. Bull. zool. Mus. Univ. Amsterdam, 1 (3) : 19-30.

Stock, J. H., 1967. A revision of the European species of the Gammarus locusta-group. Zool. Verhand., 90 : $1-56$.

-, 1968. A revision of the European species of the Echinogammarus pungens-group (Crustacea, Amphipoda). Beaufortia, 16 (211): 13-78. 